\title{
A Fast and Reliable Method to Interpret Short-Term Mortality in Perforated Peptic Ulcer: Red Cell Distribution Width is Sensitive and Specific
}

\author{
Okan Murat Akturk (D), Mikail Çakır, Yaşar Murat Vardar, Doğan Yıldırım, \\ and Muzaffer Akıncı \\ Department of Surgery, University of Health Sciences, Haseki Training and Research Hospital, Istanbul, Turkey \\ Correspondence should be addressed to Okan Murat Akturk; omakturk@gmail.com
}

Received 22 February 2021; Accepted 5 May 2021; Published 18 May 2021

Academic Editor: Christophoros Foroulis

Copyright (C) 2021 Okan Murat Akturk et al. This is an open access article distributed under the Creative Commons Attribution License, which permits unrestricted use, distribution, and reproduction in any medium, provided the original work is properly cited.

\begin{abstract}
Introduction. Peptic ulcer is an important health problem worldwide with a prevalence of around $5 \%$. Peptic ulcer perforation is a potentially mortal complication of peptic ulcer disease. We aimed to investigate the potential use of red cell distribution width as a prognostic marker in peptic ulcer perforation. Methods. The files, operation notes, biochemical and hematological parameters, and prognosis of patients who were operated for a peptic ulcer perforation were reviewed in a retrospective cohort study. The relation of red cell distribution width (RDW) to main outcome in-hospital mortality was assessed. Results. The mean age of the 172 patients was $40 \pm 17.89$. There were 158 (92\%) males and 14 (8\%) females. The in-hospital mortality was 8.7\% (15/172). The median RDW in the group with mortality was 15.00 (interquartile range (IQR): 14.30-17.20) compared with the median RDW in the group with no mortality as 13.2 (IQR: $12.80-14.00, p \leq 0.001$ ). Receiver operator characteristic curves were plotted for RDW to identify nonsurvivors and yielded a significant area under the curve as 0.812 (95\% confidence interval: $0.682-0.942$ ). The sensitivity and specificity of RDW at a cutoff value of $14.25 \%$ were calculated with an accuracy of 81.98 (95\% confidence interval: $75.40-87.41)$ as 80.00 (51.91-95.67) and 82.17 (75.27-87.81), respectively. Conclusion. Increased RDW may be of use to interpret mortality in patients with peptic ulcer perforation.
\end{abstract}

\section{Introduction}

Peptic ulcer perforation (PUP) is a serious complication of peptic ulcer disease (PUD) that causes morbidity and mortality. The lifetime prevalence of perforation in patients with PUD is reported to be around 5\% [1]. Despite advances in the treatment of PUD, perforation occurs in $2-10 \%$ of patients and accounts for more than $70 \%$ of deaths associated with the disease [2]. The onset of PUP is significant, rendering diagnosis thereof relatively easy and allowing for rapid intervention. Mortality rates are reported to be between $1.3 \%$ and 20\% [3]. Due to its relatively common occurrence, identification of patients at a greater risk of mortality at earlier stages would result in better allocation of resources regarding intensive care and transportation and earlier medical intervention.
Red cell distribution width (RDW) reflects heterogeneity among erythrocyte volumes, and elevated RDW levels indicate an increase in red blood cell size variation called anisocytosis [4]. RDW is easily obtained during routine complete blood count (CBC) lab testing, and accumulating evidence demonstrates that RDW is a valuable prognostic tool in multiple disease settings.

$\mathrm{RDW}$, as a novel inflammatory marker, is suggested to be associated with type 2 diabetes mellitus, coronary artery disease, malignant conditions, lumbar discopathies, respiratory distress syndrome, and multiple myeloma [5-10]. An elevated RDW has been associated with poorer outcomes including increased mortality in patients with ischemic heart disease, acute mesenteric ischemia, cerebrovascular disease, and renal cancer [11-14]. 
RDW is reflective of inflammation, and in the general population, a higher RDW is associated with increases in ESR and the inflammatory markers IL-6, C-reactive protein, and receptors for TNF I and II [15]. The exact mechanism underlying the link between clinical manifestations of elevated RDW and the diseases mentioned above has yet to be fully elucidated, but high levels of RDW have been associated with inflammation, poor nutritional status, and changes in erythropoiesis [16, 17]. This study investigates whether RDW can predict the prognosis of PUP patients upon hospital admission.

\section{Materials and Methods}

We evaluated the in-hospital mortality of patients receiving surgery for PUP at the General Surgery Department of Istanbul Haseki Training and Research Hospital in a retrospective cohort study. The medical records of patients who underwent emergency surgery for PUP between May 2013 and May 2017 were reviewed according to codes designated by the International Statistical Classification of Diseases and Related Health Problems, $11^{\text {th }}$ revision. This study was approved by the local ethical committee (195/26.07.2018) and conformed to the principles of the Declaration of Helsinki. Written consent to use their clinical data was obtained from patients before surgery. Preoperative diagnoses were based on clinical data and radiological findings, and chest radiogram was the most common diagnostic tool. Nearly half of the patients underwent computed abdominal tomography (CT) for differential diagnosis unless contraindicated. In addition, biochemical tests on hepatic and renal function and hemograms are routinely obtained from patients planning to undergo surgery. RDW was routinely measured as part of an automated CBC count using a hematology analyzer. The reference range of $\mathrm{RDW}$ at our hospital is $11.5-14.5 \%$. All operations were carried out under general anesthesia, either via midline incision or laparoscopy, and all patients underwent resuscitation with individualized fluid-electrolyte support therapy. Patients younger than 16 years of age or with a hematologic disorder, recent transfusion history, or recurrent perforation were excluded from the study. The primary outcome analyzed was in-hospital mortality rate.

\section{Statistical Analysis}

The main goal of this study was the prediction of mortality in the patient group. All statistics were performed using SPSS 22.0 for Windows (SPSS Inc, Chicago, IL, USA). Data were checked for normality with the Kolmogorov-Smirnov test and found to be nonnormally distributed and are thereby expressed as median and interquartile range (IQR). Categorical variables were expressed as frequencies and percentages. Patient age and sex, American Society of Anesthesiologists (ASA) physical status score, diameter of perforation area $(\mathrm{mm})$, and the following laboratory parameters were analyzed: white blood cell count (WBC; $10^{3}$ / $\left.\mathrm{mm}^{3}\right)$, RDW (\%), serum albumin $(\mathrm{g} / \mathrm{dL})$, creatinine $(\mathrm{mg} /$ $\mathrm{dL})$, total protein $(\mathrm{g} / \mathrm{dL})$, blood urea nitrogen $(\mathrm{BUN}$; $\mathrm{mg} / \mathrm{dL})$, and potassium $(\mathrm{mmol} / \mathrm{L})$. A binary logistic regression was carried out to reveal variables that were significantly associated with mortality. Fischer's exact test was used for intergroup comparisons, the Mann-Whitney $U$ test was used for nonnormally distributed continuous parameters, and Pearson's chi square test was used to analyze categorical variables. All tests were two sided, and a $p$ value of less than 0.05 was considered statistically significant. An analysis of receiver operating characteristics (ROC) curve associated with the area under the curve (AUC) was used to derive optimal cutoff values and their specificity and sensitivity to predict progression to mortality. The AUC also indicated the probability of concordance between the predicted probability of postoperative mortality and the actual postoperative state. Determinant factors were analyzed using logistic regression analysis.

\section{Results}

The median age of the 172 patients was $40 \pm 17.89$ years (range 18-93). There were 158 males (92\%) and 14 (8\%) females. Patient characteristics are provided in Table 1. All patients underwent surgery as a primary treatment. Of these, $140(81.0 \%)$ patients underwent open repair with omental patch, $17(9.7 \%)$ received laparoscopic repair with omental patch, 9 (6.0\%) underwent gastrojejunostomy, $2(1.1 \%)$ received gastrectomy with truncal vagotomy, 2 (1.1\%) had a Roux-en-Y (RNY) gastrojejunostomy, and 2 (1.1\%) had a tube duodenostomy. In two cases, appendectomy was added, as well. Fifteen patients $(8.7 \%)$ died during the 30 days following the operation. Overall median RDW was 13.3 (IQR: 12.80-14.30). The median RDW in the mortality group was 15.00 (IQR: 14.30-17.20), whereas the median RDW in the survival group was 13.20 (IQR: $12.80-14.00 ; p \leq 0.001$ ). ROC curves were plotted for RDW values to identify nonsurvivors with a significant AUC $(0.812 ; 95 \% \mathrm{CI}$ : $0.682-0.942$; Figure 1$)$. The sensitivity and specificity, positive likelihood ratio (+LR), and negative likelihood ratio (-LR) for RDW at a cutoff value of 14.25 were calculated to an accuracy of 81.98 (95\% CI: 75.40-87.41; Table 2). ROC curve analysis for ASA physical scores revealed a significant AUC of 9.11 (95\% CI: 0.87-1.00; Figure 2).

In binary regression analysis, the variables age $(p \leq 0.001)$, ASA score $(p \leq 0.001)$, WBC $(p \leq 0.001)$, albumin $(p \leq 0.001)$, total protein $(p \leq 0.001)$, diameter of perforation $(p=0.01)$, BUN $(p \leq 0.001)$, and potassium $(p \leq 0.001)$ were found to be significantly associated with mortality $(p \leq 0.001)$, but creatine was not $(p=0.17)$ (Bonferroni correction).

\section{Discussion}

PUP is a significant global health problem, constituting a surgical emergency that is potentially mortal [18]. While the prevalence of PUD has decreased globally in recent decades, this reduction has not been accompanied by a decrease in complications arising from peptic ulcers [19]. Rates of mortality following a PUP have been reported to range between $8.5 \%$ and $25.3 \%$. Thomsen et al. [20] in a 
TABLE 1: Summary of the demographic and clinical characteristics of the study group.

\begin{tabular}{lc}
\hline Parameter & Study group (IQR) \\
\hline Age (years) & $40 \pm 17.89$ (range $18-93)$ \\
Sex (female/male) & $14 / 158(8 \%$ vs. $92 \%)$ \\
Creatinine $(\mathrm{mg} / \mathrm{dL})$ & 0.83 (IQR: $0.69-1.04)$ \\
WBC $\left(10^{3} / \mathrm{mm}^{3}\right)$ & 13.21 (IQR: $10.65-17.54)$ \\
RDW $(\%)$ & 13.3 (IQR: $12.80-14.30)$ \\
Total protein $(\mathrm{g} / \mathrm{dL})$ & 6.98 (IQR: $6.50-7.41)$ \\
Albumin $(\mathrm{g} / \mathrm{dL})$ & 4.15 (IQR: $3.48-4.40)$ \\
Perforation diameter $(\mathrm{mm})$ & 5 (IQR: $5.00-10.00)$ \\
Hospital stay (days) & 5 (IQR: $5.00-7.00)$ \\
\hline
\end{tabular}

Data are presented as median value (interquartile range). IQR, interquartile range.

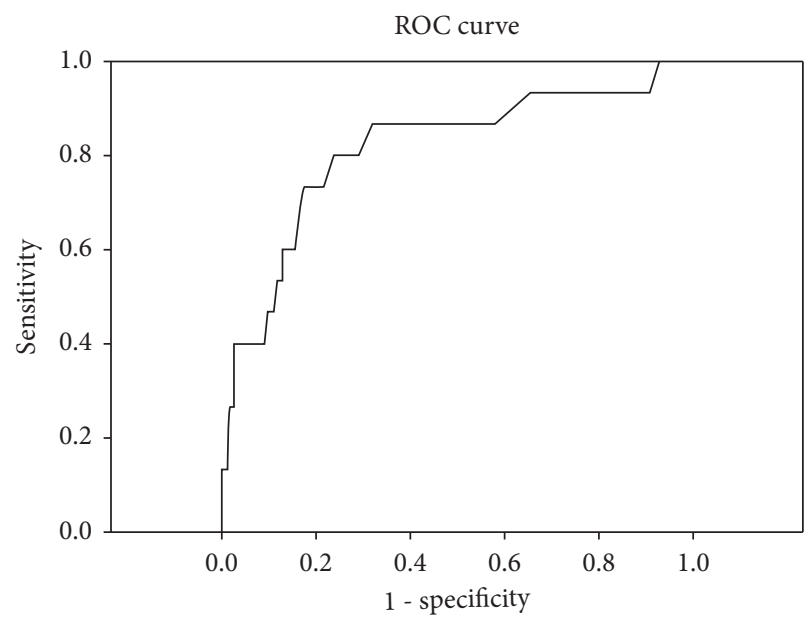

FIGURE 1: Receiver operating characteristic (ROC) curve analysis for RDW to identify nonsurvivors.

Area under the curve (AUC) is 0.812 (95\% CI: 0.682-0.942).

retrospective cohort of 2,061 patients reported a mortality rate of 25.3\%; Noguiera et al. [21] reported a mortality rate of $10 \%$ in 210 patients; and two Turkish studies reported mortality of $8.8 \%$ and $8.6 \%[22,23]$. In our study, the inhospital mortality rate was found to be $8.7 \%$, which closely aligns with results from the Turkish studies.

There are several classification systems for predicting severity of disease in PUD patients. The Boey score was the first to be developed and remains popular for predicting mortality in PUP using three major variables: major medical illness, preoperative shock, and a perforation duration greater than 24 hours [24]. Boey score was able to correctly predict mortality in 93.8\% of PUP patients in a prospective study [25].

Another system for prediction of short-term mortality is the peptic ulcer perforation (PULP) score, a method that evolved from a multicenter study on 2,668 patients in Denmark. The PULP comprises 8 variables associated with poor prognosis: age over 65 , active malignant disease or acquired immunodeficiency syndrome, liver cirrhosis, steroid use, length of time elapsed between perforation and admission, preoperative shock, raised serum creatinine level, and an ASA grade over I [26]. The AUC for the PULP and Boey scores was also calculated, with results of 0.83 and 0.70 , respectively.
While the ASA assessment provides a general score for surgery suitability, it is also a commonly used risk score for PUP. In our study, the AUC for ASA was 0.912, similar to results from a 152 patient study by Lohsiriwat et al. [27], which reported a mortality rate of $9 \%$ and an AUC of 0.91. In another study of 117 patients, Buck et al. [28] reported an AUC for ASA in patients with PUP of 0.73 and a mortality rate of $17 \%$. Moller et al. [29] studied ASA scores in a group of 708 patients and found an AUC of 0.78 with a mortality rate of $27 \%$. ASA is an assessment of overall patient health based on five signifiers, and mortality risk increases with higher values [30]. However, the ASA score has been reported to have interobserver variability [31].

PULP combines properties introduced by the Boey score and PULP, scoring slightly better than both systems in the prediction of mortality [29]. The Boey score was developed using American patients in the early 1980s, while PULP is a more recently derived classification system (Table 3).

Significant risk factors which may cause death are the presence of shock at admission, comorbidities, resection surgery, female, elderly patients, a delay in presentation of more than $24 \mathrm{~h}$, metabolic acidosis, acute renal failure, hypoalbuminemia, being underweight, and smokers [3].

RDW was found to be strongly associated with all causes of mortality in both middle-aged and elderly adults in a large cohort study, and mortality rates were particularly elevated in patients with an RDW greater than 13.4; for every $1 \%$ increase in RDW, mortality risk increased by $22 \%$ [32]. The underlying mechanisms associated with RDW elevation are as yet not fully understood. Erythropoietin in serum increases along with aging to compensate for subclinical blood loss, increased red blood cell turnover, or increased erythropoietin resistance in red cell precursors [33]. This is thought to lead to an overall higher RDW in older patients. Erythropoietin itself might also be partially responsible for changes in red cell volume and contribute to the predictive qualities of RDW.

RDW has been shown to be associated with mortality in general, in addition to risk of death from cardiovascular disease, cancer, and chronic lower respiratory tract disease, even after adjusting for anemia and related nutritional deficiencies in outpatients [34]. Lippi et al. [35] reported a graded association of RDW with high-sensitivity C-reactive protein and erythrocyte sedimentation rate, independent of several confounding factors.

Given that an elevated RDW is associated with comorbidity and inflammatory status, it alone might substitute for scoring systems that incorporate patient fitness, such as ASA score or inflammation. Moreover, the time lapse between the onset of symptoms and hospital admission may lead to higher inflammatory status in cases of delayed intervention.

In this study, we suggest that RDW is a specific and sensitive laboratory marker that can be used to predict mortality in patients with PUP. As far as we know, this is the first study evaluating RDW for prediction of PUP patient mortality. 
TABLE 2: Sensitivity, specificity, positive likelihood ratio (+LR), negative likelihood ratio (LR), positive predictive value, and negative predictive value of RDW for mortality at the optimal cutoff level.

\begin{tabular}{llllll}
\hline RDW & Sensitivity (95\% CI) & Specificity (95\% CI) & +LR & -LR & Positive predictive value (95\% CI) Negative predictive value (95\% CI) \\
\hline
\end{tabular}

\begin{tabular}{lllllll}
\hline$>14.25$ & $80.00(51.91-95.67)$ & $82.17(75.27-87.81)$ & 4.49 & 0.24 & $30.00(21.96-39.49)$ & 97.73 (93.97-99.16)
\end{tabular}

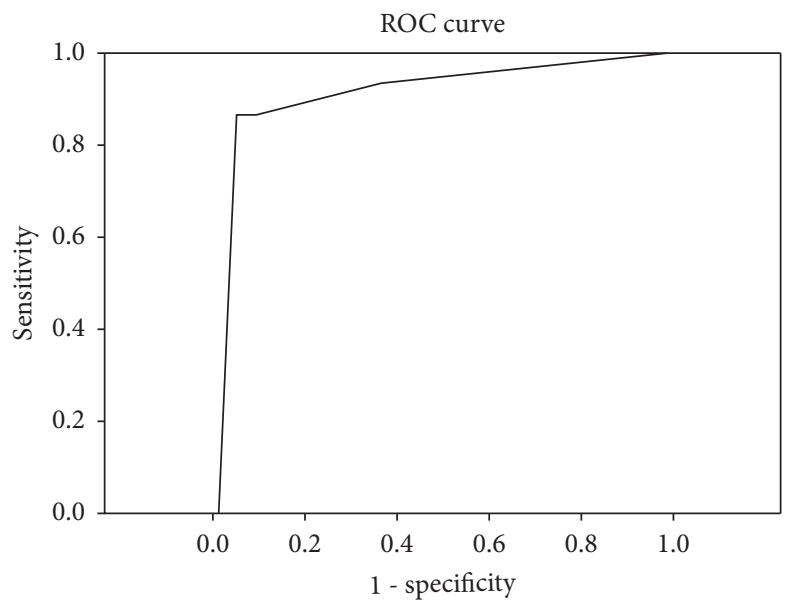

FIGURE 2: Receiver operating characteristic (ROC) curve analysis for ASA physical scores.

Receiver operating characteristic (ROC) curve analysis for ASA physical scores revealed a significant AUC of 9.11 (95\% CI: 0.87-1.00).

TABLE 3: Overview of predictive systems.

\begin{tabular}{lccc}
\hline $\begin{array}{l}\text { Boey, } \\
1987\end{array}$ & Patients with PUP & 30 day mortality & Presentation within or after 24 hours; shock; level of comorbidity. \\
\hline $\begin{array}{l}\text { PULP, } \\
2012\end{array}$ & Patients with PUP & 30 day mortality & $\begin{array}{c}\text { Presentation within or after 24 hours; shock; ASA score, active } \\
\text { malignancy, liver insufficiency; serum creatinine }>130 \text { mmol/L; acquired } \\
\text { immune deficiency syndrome }\end{array}$ \\
\hline $\begin{array}{l}\text { ASA, } \\
1941\end{array}$ & $\begin{array}{c}\text { General surgical } \\
\text { population }\end{array}$ & $\begin{array}{c}\text { Preoperative risk assessment } \\
\text { for surgical patients }\end{array}$ & Degree of comorbidity and systemic disease \\
\hline
\end{tabular}

There are certain limitations to our study, the first of which being that it is a retrospective study. Also, as it is not a multicenter study, selection bias is inevitable. Our study population may be younger than PUP sufferers in general, as well, because our hospital serves a large population of foreignborn residents who may have lacked adequate previous medical care. Our study reflects a low positive predictivity, although a very sensitive one, potentially because of the relatively low prevalence of the studied variable: mortality. Besides, it is not proposed as a substitute for available risk scoring systems but provides clear foresight for the surgeon; thus, this field needs additional studies.

\section{Conclusion}

Increased RDW in preoperative assessment may be a sensitive and specific indicator of mortality likelihood in patients with PUP. Surgeons can employ elevated RDW as a prognostic factor and should carefully monitor patients with increased RDW values.

\section{Data Availability}

The data used to support the findings of this study are available from the corresponding author upon reasonable request with the permission of the institute.

\section{Conflicts of Interest}

The authors declare that there are no conflicts of interest.

\section{References}

[1] D. Vaira, M. Menegatti, and M. Miglioli, "What is the role of Helicobacter pylori in complicated ulcer disease?" Gastroenterology, vol. 113, pp. 78-84, 1997.

[2] M. J. O. E. Bertleff and J. F. Lange, "Perforated peptic ulcer disease: a review of history and treatment," Digestive Surgery, vol. 27, no. 3, pp. 161-169, 2010.

[3] K. T. Chung and V. G. Shelat, "Perforated peptic ulcer-an update," World Journal of Gastrointestinal Surgery, vol. 9, no. 1, pp. 1-12, 2017. 
[4] H. Abdullah, Y. Sim, Y. T. Sim et al., "Preoperative Red Cell Distribution Width and 30-day mortality in older patients undergoing non-cardiac surgery: a retrospective cohort observational study," Scientific Reports, vol. 8, p. 6226, 2018.

[5] Y. Wang, P. Yang, Z Yan et al., "The relationship between erythrocytes and Diabetes Mellitus," Journal of Diabetes Research, vol. 2021, Article ID 6656062, 9 pages, 2021.

[6] K. Bujak, J. Wasilewski, T. Osadnik, S Jonczyk, A. Kołodziejska, and M. Gierlotka, "The prognostic role of red blood cell distribution width in coronary artery disease: a review of the pathophysiology," Disease Markers, vol. 2015, Article ID 824624, 12 pages, 2015.

[7] C. Yüksel, O. Erşen, S. Culcu, B. Bakırarar, A. E. Unal, and S. Demirci, "Prognostic role of red distribution width (RDW) value in gastric cancer," Journal of the College of Physicians and Surgeons of Pakistan, vol. 31, pp. 21-26, 2021.

[8] Y. Dagistan, E. Dagistan, A. R. Gezici et al., "Could red cell distribution width and mean platelet volume be a predictor for lumbar disc hernias?" Ideggyogy Sz, vol. 69, pp. 411-414, 2016.

[9] A. Alkhatib, L. L. Price, R. Esteitie, and P. LaCamera, A predictive model for acute respiratory distress syndrome mortality using red cell distribution width," Critical Care Research and Practice, vol. 2020, Article ID 3832683, 6 pages, 2020.

[10] H. Lee, S. Y. Kong, J. Y. Sohn, H. Shim, H. S. Youn, and S. Lee, "Elevated red blood cell distribution width as a simple prognostic factor in patients with symptomatic multiple myeloma," BioMed Research International, vol. 2014, Article ID 145619, 8 pages, 2014.

[11] İ. Bilgiç, F. Dolu, K. Şenol, and M. Tez, "Prognostic significance of red cell distribution width in acute mesenteric ischemia," Perfusion, vol. 30, no. 2, pp. 161-165, 2015.

[12] G. M. Felker, L. A. Allen, S. J. Pocock et al., "Red cell distribution width as a novel prognostic marker in heart failure," Journal of the American College of Cardiology, vol. 50, no. 1, pp. 40-47, 2007.

[13] J. Kim, Y. Kim, T.-J. Song et al., "Red blood cell distribution width is associated with poor clinical outcome in acute cerebral infarction," Thrombosis and Haemostasis, vol. 108, no. 08, pp. 349-356, 2012.

[14] F.-M. Wang, G. Xu, Y. Zhang, and L.-L. Ma, "Red cell distribution width is associated with presence, stage, and grade in patients with renal cell carcinoma," Disease Markers, vol. 2014, Article ID 860419, 7 pages, 2014.

[15] H. S. Bazick, D. Chang, K. Mahadevappa, F. K. Gibbons, and K. B. Christopher, "Red cell distribution width and all-cause mortality in critically ill patients," Critical Care Medicine, vol. 39, no. 8, pp. 1913-1921, 2011.

[16] A. Vayá, A. Sarnago, O. Fuster, R. Alis, and M. Romagnoli, "Influence of inflammatory and lipidic parameters on red blood cell distribution width in a healthy population," Clinical Hemorheology and Microcirculation, vol. 59, no. 4, pp. 379-385, 2015.

[17] L. Ferrucci, J. M. Guralnik, R. C. Woodman et al., "Proinflammatory state and circulating erythropoietin in persons with and without anemia," American Journal of Medicine, vol. 118, p. 1288, 2005.

[18] C. Svanes, "Trends in perforated peptic ulcer: incidence, etiology, treatment, and prognosis," World Journal of Surgery, vol. 24 , no. 3, pp. 277-283, 2000.

[19] K. Søreide, K. Thorsen, and J. A. Søreide, "Strategies to improve the outcome of emergency surgery for perforated peptic ulcer," British Journal of Surgery, vol. 101, no. 1, pp. e51-e64, 2013.

[20] R. W. Thomsen, A. Riis, S. Christensen, M. Norgaard, and H. T. Sorensen, "Diabetes and 30-day mortality from peptic ulcer bleeding and perforation: a Danish population-based cohort study," Diabetes Care, vol. 29, no. 4, pp. 805-810, 2006.

[21] C. Noguiera, A. S. Silva, J. N. Santos et al., "Perforated peptic ulcer: main factors of morbidity and mortality," World Journal of Surgery, vol. 27, no. 7, pp. 782-787, 2003.

[22] N. Ozalp, B. Zulfikaroglu, I. Bilgic, and M. Koc, "Evaluation of risk factors for mortality in perforated peptic ulcer in Ankara Numune Teaching Hospital, Ankara, Turkey," East African Medical Journal, vol. 81, pp. 634-637, 2005.

[23] B. Kocer, S. Surmeli, C. Solak et al., "Factors affecting mortality and morbidity in patients with peptic ulcer perforation," Journal of Gastroenterology and Hepatology, vol. 22, no. 4, pp. 565-570, 2007.

[24] J. Boey, J. Wong, and G. B. Ong, "A prospective study of operative risk factors in perforated duodenal ulcers," Annals of Surgery, vol. 195, no. 3, pp. 265-269, 1982.

[25] J. Boey, "Risk stratification in perforated duodenal ulcers. a prospective validation of predictive factors," Annals of Surgery, vol. 205, pp. 22-26, 2000.

[26] M. H. Moller, M. C. Engebjerg, S. Adamsen, J. Bendix, and R. W. Thomsen, "The peptic ulcer perforation (PULP) score: a predictor of mortality following peptic ulcer perforation. a cohort study," Acta Anaesthesiologica Scandinavica, vol. 56, pp. 655-662, 2012.

[27] V. Lohsiriwat, S. Prapasrivorakul, and D. Lohsiriwat, "Perforated peptic ulcer: clinical presentation, surgical outcomes, and the accuracy of the Boey scoring system in predicting postoperative morbidity and mortality," World Journal of Surgery, vol. 33, no. 1, pp. 80-85, 2008.

[28] D. L. Buck, M. Vester-Andersen, and M. H. Møller, “Accuracy of clinical prediction rules in peptic ulcer perforation: an observational study," Scandinavian Journal of Gastroenterology, vol. 47, no. 1, pp. 28-35, 2012.

[29] M. H. Møller, S. Adamsen, R. W. Thomsen, A. M. Møller, and Peptic Ulcer Perforation (PULP) Trial Group, "Multicentre trial of a perioperative protocol to reduce mortality in patients with peptic ulcer perforation," British Journal of Surgery, vol. 98, pp. 802-810, 2011.

[30] M. Daabiss, "American Society of Anaesthesiologists physical status classification," Indian Journal of Anaesthesia, vol. 55, no. 2, pp. 111-115, 2011.

[31] P. H. K. Mak, R. C. H. Campbell, M. G. Irwin, and American Society of Anesthesiologists, "The ASA physical status classification: inter-observer consistency," Anaesthesia and Intensive Care, vol. 30, no. 5, pp. 633-640, 2002.

[32] K. V. Patel, L. Ferrucci, W. B. Ershler, D. L. Longo, and J. M. Guralnik, "Red blood cell distribution width and the risk of death in middle-aged and older adults," Archives of Internal Medicine, vol. 169, no. 5, p. 515, 2009.

[33] W. B. Ershler, S. Sheng, J. McKelvey et al., "Serum erythropoietin and aging: a longitudinal analysis," Journal of the American Geriatrics Society, vol. 53, no. 8, pp. 1360-1365, 2005.

[34] T. S. Perlstein, J. Weuve, M. A. Pfeffer, and J. A. Beckman, "Red blood cell distribution width and mortality risk in a community-based prospective cohort," Archives of Internal Medicine, vol. 169, no. 6, pp. 588-594, 2009.

[35] G. Lippi, G. Targher, M. Montagnana, G. L. Salvagno, G. Zoppini, and G. C. Guidi, "Relationship between red blood cell distribution width and kidney function tests in a large cohort of unselected outpatients," Scandinavian Journal of Clinical and Laboratory Investigation, vol. 68, no. 8, pp. $745-748,2008$. 